University of Wollongong

Research Online

Faculty of Engineering and Information

Faculty of Engineering and Information

Sciences - Papers: Part A

Sciences

2016

\title{
ASL-incorporated pharmacokinetic modelling of PET data with reduced acquisition time: Application to amyloid imaging
}

Catherine L. Scott

University College London, cscott@uow.edu.au

Jieqing Jiao

University College London

Andrew Melbourne

University College London

Jonathan M. Schott

University College London

Brian F. Hutton

University of Wollongong, b.hutton@ucl.ac.uk

See next page for additional authors

Follow this and additional works at: https://ro.uow.edu.au/eispapers

Part of the Engineering Commons, and the Science and Technology Studies Commons

Research Online is the open access institutional repository for the University of Wollongong. For further information contact the UOW Library: research-pubs@uow.edu.au 


\title{
ASL-incorporated pharmacokinetic modelling of PET data with reduced acquisition time: Application to amyloid imaging
}

\author{
Abstract \\ Pharmacokinetic analysis of Positron Emission Tomography (PET) data typically requires at least one \\ hour of image acquisition, which poses a great disadvantage in clinical practice. In this work, we propose \\ a novel approach for pharmacokinetic modelling with significantly reduced PET acquisition time, by \\ incorporating the blood flow information from simultaneously acquired arterial spin labelling (ASL) \\ magnetic resonance imaging (MRI). A relationship is established between blood flow, measured by ASL, \\ and the transfer rate constant from plasma to tissue of the PET tracer, leading to modified PET kinetic \\ models with ASL-derived flow information. Evaluation on clinical amyloid imaging data from an \\ Alzheimer's disease (AD) study shows that the proposed approach with the simplified reference tissue \\ model can achieve amyloid burden estimation from $30 \mathrm{~min}\left[{ }^{18} \mathrm{~F}\right]$ florbetapir PET data and $5 \mathrm{~min}$ \\ simultaneous ASL MR data, which is comparable with the estimation from 60 min PET data (mean \\ error $=-0.03)$. Conversely, standardised uptake value ratio (SUVR), the alternative measure from the data \\ showed a positive bias in areas of higher amyloid burden (mean error=0.07). \\ Disciplines \\ Engineering | Science and Technology Studies

\section{Publication Details} \\ Scott, C. J., Jiao, J., Melbourne, A., Schott, J. M., Hutton, B. F. \& Ourselin, S. (2016). ASL-incorporated \\ pharmacokinetic modelling of PET data with reduced acquisition time: Application to amyloid imaging. \\ Lecture Notes in Computer Science, 9902 406-413.

\section{Authors} \\ Catherine L. Scott, Jieqing Jiao, Andrew Melbourne, Jonathan M. Schott, Brian F. Hutton, and Sebastien \\ Ourselin
}




\title{
ASL-incorporated Pharmacokinetic Modelling of PET Data with Reduced Acquisition Time: Application to Amyloid Imaging
}

\author{
Catherine J Scott ${ }^{1}$, Jieqing Jiao ${ }^{1}$, Andrew Melbourne ${ }^{1}$, Jonathan M Schott ${ }^{2}$, \\ Brian F Hutton ${ }^{3,4}$, and Sébastien Ourselin ${ }^{1,2}$

\footnotetext{
${ }^{1}$ Translational Imaging Group, CMIC, University College London, London, UK ${ }^{3}$ Institute of Nuclear Medicine, University College London, London, UK
} \\ ${ }^{2}$ Dementia Research Centre, Institute of Neurology, University College London, UK \\ ${ }^{4}$ Centre for Medical Radiation Physics, University of Wollongong, NSW, Australia
}

\begin{abstract}
Pharmacokinetic analysis of Positron Emission Tomography (PET) data typically requires at least one hour of image acquisition, which poses a great disadvantage in clinical practice. In this work, we propose a novel approach for pharmacokinetic modelling with significantly reduced PET acquisition time, by incorporating the blood flow information from simultaneously acquired arterial spin labelling (ASL) magnetic resonance imaging (MRI). A relationship is established between blood flow, measured by ASL, and the transfer rate constant from plasma to tissue of the PET tracer, leading to modified PET kinetic models with ASL-derived flow information. Evaluation on clinical amyloid imaging data from an Alzheimer's disease study shows that the proposed approach with the simplified reference tissue model can achieve amyloid burden estimation from 30-min $\left.{ }^{18} \mathrm{~F}\right]$ florbetapir PET data and 5-min simultaneous ASL MR data, which is comparable with the estimation from 60 -min PET data (mean error $=-0.03$ ). Conversely, standardised uptake value ratio (SUVR), the alternative measure from the data showed a positive bias in areas of higher amyloid burden (mean error $=0.07$ ).
\end{abstract}

\section{Introduction}

Position Emission Tomography (PET) is currently the most sensitive in vivo molecular imaging technique to provide a non-invasive assay of the human body. Dynamic PET image data acquired following the injection of a radioactive tracer allows the use of pharmacokinetic modelling techniques to quantify a range of biological, physiological and biochemical parameters. However, a typical dynamic PET scan requires at least 1 hour to sufficiently cover the underlying processes. The long scan duration is prohibitive for routine clinical use, where time is limited, and data integrity is risked by the increased chance of subject motion.

Currently clinical imaging in PET is often performed using single time point estimates (static imaging) of tracer uptake, such as the standardised uptake value ratio (SUVR). SUVR, a semi-quantitative measure of uptake, is the ratio of the activity concentration within a region relative to the concentration 
in a tissue which is free from the imaging target, called the reference region. It usually requires 10 mins of PET data, which are acquired once non-specifically bound tracer reaches equilibrium between a region and the reference tissue, approximately 50 mins or more post injection. This measure is expected to correlate with fully quantitative estimates derived from the full dynamic PET data. However, changes in blood flow affect the delivery of the tracer to tissue and consequently alter the tracer concentration in the tissue when a static image is acquired. Without the blood flow information contained in the early dynamic data, there is no way to account for the influence of the changes in blood flow, thus SUVR values can be biased. This has been highlighted in longitudinal studies, where pathophysiological changes in blood flow have caused spurious changes in SUVR values which do not reflect imaging target abundance. [1]. The estimates derived by kinetic modelling are not biased in this way, as the full dynamic curve contains blood flow information, which is parametrised within the model.

In neuroimaging, cerebral blood flow can be measured using arterial spin labelled (ASL) MRI, where magnetically tagged blood is used as an endogenous contrast agent. With the advent of PET-MRI scanners, this information can be acquired concurrently with PET data. Therefore the blood flow information from the ASL can be used in pharmacokinetic analysis when the early part of a dynamic PET scan, which involves blood flow, is not collected. This will lead to the reduction of PET acquisition time needed to perform pharmacokinetic modelling.

In this work, we propose a novel approach for combining PET and ASL information to derive the parameters of interest with a greatly reduced scanning time, Figure 1. To our knowledge,

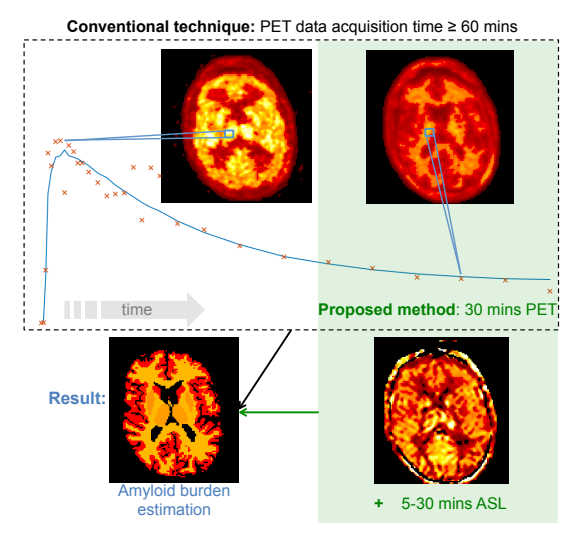

Fig. 1: Dynamic PET acquisition for amyloid burden quantification and time reduction for the proposed method. this is the first time that ASL blood flow estimates have been used to perform PET kinetic analysis to reduce image acquisition time. We evaluated the proposed approach in an AD study using $\left[{ }^{18} \mathrm{~F}\right]$ florbetapir, a PET radiotracer that binds to amyloid- $\beta$, which is considered to be an important target in the $\mathrm{AD}$ brain.

\section{Methods}

\subsection{CBF estimation with ASL MRI}

The cerebral blood flow (CBF) map is estimated from pseudo continuous arterial spin labelling (PCASL) data using the relationship established in [2]. The 
parameter values used in this work were $0.9 \mathrm{ml} / \mathrm{g}$ for the plasma/tissue partition coefficient, a blood $T 1$ value of $1650 \mathrm{~ms}$, and a labelling efficiency of 0.85 .

\section{$2.2 \quad$ Amyloid- $\beta$ burden estimation with SRTM}

In this work, the simplified reference tissue model (SRTM) [3] was used to quantify the PET data. SRTM describes the tracer-target interaction using a single tissue compartment model. Using the tracer time activity curve in the reference region $C_{R}(t)$ as an input function, the operational equation between the tracer time activity curve in the target tissue $C_{T}(t)$ and $C_{R}(t)$ is formulated as:

$$
C_{T}(t)=R_{1} C_{R}(t)+\left(k_{2}-R_{1} \frac{k_{2}}{1+B P_{N D}}\right) C_{R}(t) \otimes e^{-\frac{k_{2}}{1+B P_{N D}} t},
$$

where $t$ denotes time and $t=0$ at tracer injection, $R_{1}$ is the local rate of delivery in the target tissue relative to reference tissue, $k_{2}$ is the rate constant from target tissue to blood, $B P_{N D}$ is the binding potential that is proportional to the density of amyloid- $\beta$, and $\otimes$ denotes the convolution operator. Cerebellar grey matter is used as the reference region to derive $C_{R}(t)$ as it is considered to be devoid of amyloid- $\beta$ in this study [4]. $B P_{N D}$, as the outcome measure of interest to represent the amyloid- $\beta$ burden, can then be estimated together with $R_{1}$ and $k_{2}$ by performing curve-fitting using (1) with $C_{T}(t)$ and $C_{R}(t)$ extracted from PET data acquired from tracer injection over a sufficient duration. We used a linearised version of SRTM [5] to calculate $B P_{N D}, R_{1}$ and $k_{2}$ from dynamic PET data of 0:60 mins as the gold standard.

\subsection{SRTM with incomplete PET scan and CBF}

Population-based extrapolation of reference input $C_{R}(t)$ To estimate $B P_{N D}$ using the PET data where the early part from the tracer injection is absent $\left(t \in\left[t_{s}, t_{e}\right], t_{s}>0\right)$, firstly extrapolation is required to have the reference input $C_{R}(t)$ for $t \in\left[0, t_{s}\right]$ so that the convolution term in (1) can be calculated. In this work the whole reference input $C_{R}(t)$ for $t \in\left[0, t_{e}\right]$ was generated using a single tissue compartment model $C_{R}(t)=K_{1}^{\prime} e^{-k_{2}^{\prime} t} \otimes \alpha A I F(t)$. If we assume $A I F(t)$, $t \in\left[0, t_{e}\right]$ is a population arterial input function with $\alpha$ being an individual scaling factor, $k_{2}^{\prime}$ a population rate constant from reference tissue to blood, and $K_{1}^{\prime}$ an individual rate constant from blood to reference tissue, then $K_{1}^{\prime} \alpha$ can be estimated by scaling a measured population-based reference input curve $C_{R}^{p}(t)$, $t \in\left[0, t_{e}\right]$ to match the individual $C_{R}(t), t \in\left[t_{s}, t_{e}\right]$ to generate $C_{R}(t), t \in\left[0, t_{e}\right]$.

$\boldsymbol{R}_{\mathbf{1}}$ estimation with CBF ASL is used to measure the CBF, flow denoted by $F$, which is converted into a pseudo $R_{1}$ estimate to use in SRTM. $R_{1}$ is defined as $R_{1}=K_{1} / K_{1}^{\prime}$ where $K_{1}$ is the rate constant from blood to target tissue and $K_{1}^{\prime}$ is the rate constant from blood to reference tissue.

Based on the Renkin-Crone model, the relationship between $K_{1}$ and $F$ can be described as

$$
K_{1}=E F=\left(1-e^{-\frac{P S}{F}}\right) F,
$$


where $E$ denotes the net extraction, $P$ is the vessel permeability and $S$ the surface area. Under common physiological conditions of flow, where $P S$ is high $(>3 \mathrm{ml}$. $\left.100 \mathrm{~g}^{-1} \cdot \mathrm{min}^{-1}\right)$, the relationship between $K_{1}$ and flow $F$ is linear. In the absence of knowledge on $P S$ across the brain, we assume that it is sufficiently high such that the relationship between $K_{1}$ and $F$, and in turn the relationship between $R_{1}$ and $F$, can be approximated as a linear function. Linear regression between $R_{1}$ and $F$ was performed on a group of subjects, and the linear relationship was then applied to a different group of subjects to convert CBF to a pseudo $R_{1}$ value for estimating $B P_{N D}$ with incomplete PET data.

SRTM with CBF-derived $\boldsymbol{R}_{1}$ and extrapolated $\boldsymbol{C}_{\boldsymbol{R}}(\boldsymbol{t})$ Rewrite (1) as $C_{T}^{\prime}(t)=\phi C_{R}(t) \otimes e^{-\theta t}$, where $C_{T}^{\prime}(t)=C_{T}(t)-R_{1} C_{R}(t)$ is calculated from the $C_{T}(t)$ and $C_{R}(t)$ extracted from the measured PET data for $t \in\left[t_{s}, t_{e}\right]$, and $R_{1}$ is derived from the CBF. Here, $\phi=k_{2}-R_{1} k_{2} /\left(1+B P_{N D}\right)$ and $\theta=k_{2} /\left(1+B P_{N D}\right)$ are unknown. To solve $\phi$ and $\theta$, we used the basis functions defined in [5] to precalculate the convolution term using the extrapolated $C_{R}(t), t \in\left[0, t_{e}\right]$ with a range of biologically plausible values for $\theta . B P_{N D}$ and $k_{2}$ are then derived from $\phi, \theta$ and the CBF-derived $R_{1}$.

\section{Experiments and Results}

Data We evaluated the proposed method on data from 11 cognitively normal subjects participating in Insight 46, a neuroimaging sub-study of the MRC National Survey of Health and Development, who underwent amyloid PET and multi-modal MR imaging on a Siemens Biograph mMR PET/MR scanner. List mode PET data were acquired for 60 mins following intravenous injection of $\left[{ }^{18} \mathrm{~F}\right]$ florbetapir, a radiotracer that binds to amyloid- $\beta$. For PET image reconstruction, simultaneously acquired structural MR was used to synthesise CT data and calculate the $\mu$-map [6]. Dynamic PET data were binned into $15 \mathrm{~s} \times 4$, $30 s \times 8,60 s \times 9,180 s \times 2,300 s \times 8$ time frames, and reconstructed using the manufacturer's software with corrections for dead-time, attenuation, scatter (based on the synthesised CT), randoms and normalisation. PCASL data were acquired using a 3D GRASE readout with voxels of $1.88 \times 1.88 \times 4 \mathrm{~mm} .10$ control-label pairs were acquired with a pulse duration and post labelling delay of $1800 \mathrm{~ms}$.

Data Processing Framework T1-weighted images were parcellated [7] into amygdala, pons, brainstem, cerebellum (white and grey separately), hippocampus, cerebral white matter, putamen, thalamus and 6 cortical grey matter regions, with left and right hemispheres combined. The T1-weighted image was rigidly registered to both ASL and PET space, and the transformation was propagated to the parcellation. Regional average $\mathrm{CBF}$ values were calculated, and the PET time activity curves were averaged across the region prior to kinetic modelling. PET data acquired during 30:60 mins were used to evaluate the proposed method. To estimate the reference region activity in the missing 
time frames, a population averaged reference input was extracted from 14 age matched subjects with 60 -mins $\left[{ }^{18} \mathrm{~F}\right]$ florbetapir PET data. To establish the relationship between the $\mathrm{CBF}$ and $R_{1}$ values, linear regression was performed on data from 5 subjects and the proposed approach was tested on the remaining 6 subjects. A summary of the data processing framework is shown in Figure 2.

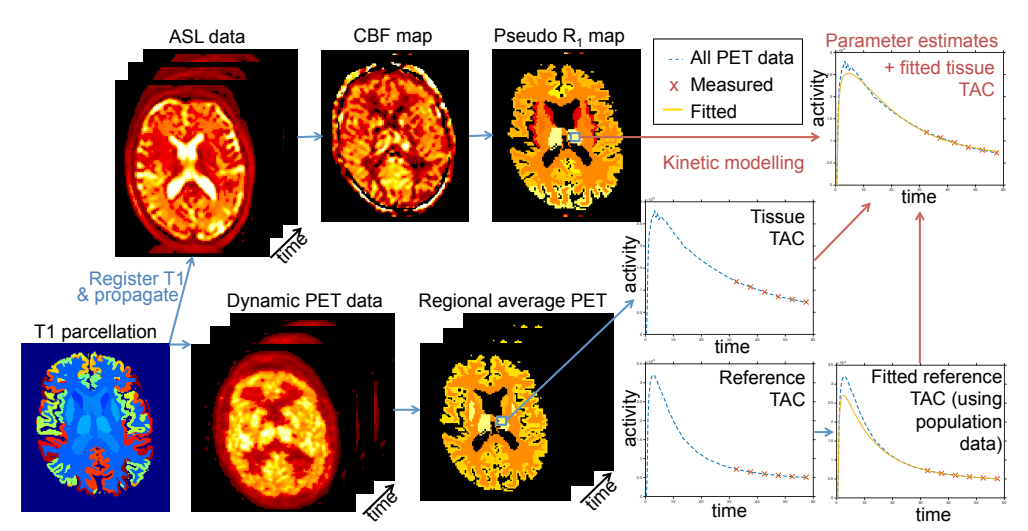

Fig. 2: Overview- parcellation is registered to PET and ASL to calculate regional average values. ASL data is converted into CBF-derived $R_{1}$ values using the linear regression relationship. A population reference tissue time activity curve of 0:60 mins combined with the measured reference tissue data (30:60 mins) is used with the CBF-derived $R_{1}$ and the measured PET tissue data (30:60 mins), to apply the modified simplified reference tissue model to estimate $B P_{N D}$.

\subsection{Comparison of proposed method with gold standard}

Figure 3a shows $B P_{N D}$, the measure of the amyloid burden, estimated using the proposed method with 30:60 mins data plotted against the gold standard using the full 60-min dynamic data. Linear regression of all subjects and regions shows that the proposed method offers a good approximation of the gold standard as it closely follows the line of identity (blue dashed line), which is within the $95 \%$ confidence interval (CI) of the regression (shaded area). Furthermore, subject specific Pearson correlation coefficients, $\rho$, show a high linear correlation.

The alternative measure used in clinical practice, $S U V R-1$, was calculated from PET data over 50:60 mins for comparison, Figure 3b. Whilst $\rho$ is still high for each subject, a clear bias is shown as $S U V R-1$ overestimates the binding potential at higher values. The mean error quantifies the bias between the estimates and the gold standard which is 0.0740 for $S U V R-1$, indicative of the systematic overestimation, compared to -0.0311 for the proposed method. The proposed method also has a lower mean square error (0.0151 compared to 


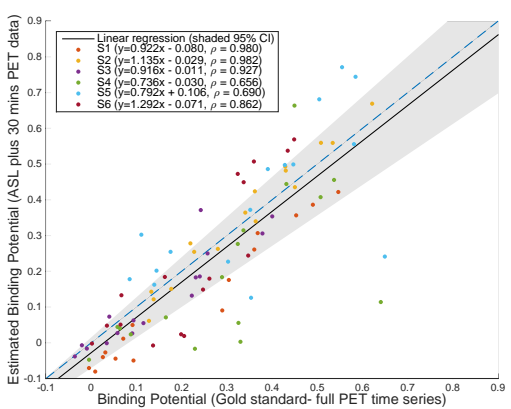

(a) Proposed method

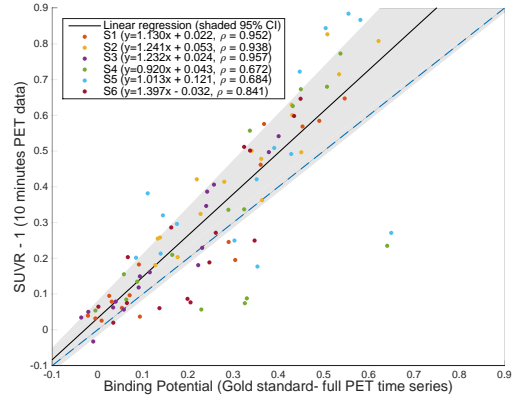

(b) $S U V R-1$

Fig. 3: Estimated amyloid burden against the gold standard value calculated using full PET time series.

0.0247 for $S U V R-1)$, and variance (0.0142 compared to 0.0194 for $S U V R-1)$. Figure 4 shows $B P_{N D}$ maps for a subject, comparing the gold standard with the proposed method and $S U V R-1$ regionally. The proposed method shows good agreement with the gold standard, with slight overestimation of the cortical white matter. For the $S U V R-1$ estimation, amyloid burden is greatly overestimated within both grey and white matter structures. The difference map shows that the errors in the proposed method are far lower than for $S U V R-1$.
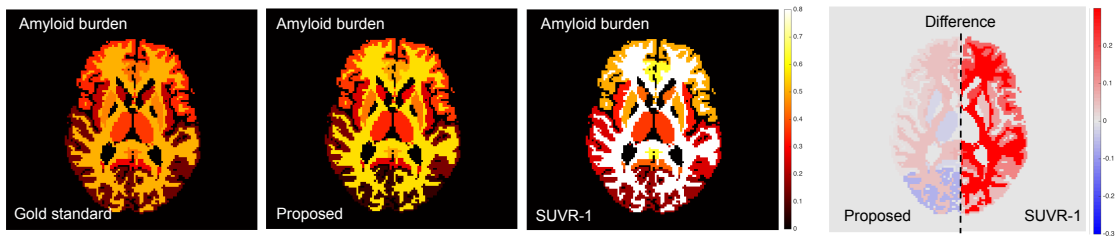

Fig. 4: Regional average binding potential maps for (left to right) gold standard, proposed method, $S U V R-1$, difference maps compared to gold standard.

\subsection{Influence of $R_{1}$ estimation on amyloid quantification}

Whilst Figure 3a demonstrates a high similarity between binding potential estimation using the gold standard and the proposed method, there is a noise component which introduces variation around the line of identity. This is due to noise in the PET data, noise in the CBF-derived $R_{1}$ estimate from the ASL data, and inaccuracies in the estimation of the reference tissue input. 
To demonstrate the influence of the CBF-derived $R_{1}$ estimate using ASL data, the proposed method was applied using the $R_{1}$ estimated using the gold standard technique instead of the CBF-derived $R_{1}$. The population input function and 30:60 mins PET data were used as before. This represents the optimal case in which $R_{1}$ can be determined exactly from the ASL data. Figure 5a shows that the variance in the binding potential estimate has been reduced (from 0.0142 to 0.008 ), and the linear regression line lies along identity with a narrow CI. This is expected since the CBF map from the ASL is noisy, and linear regression performed to determine the relationship between $\mathrm{CBF}$ and $R_{1}$ was performed with only 5 subjects, and therefore may not be generalisable. However, for the data used in this study the estimation of $R_{1}$ from CBF is sufficiently accurate that the $B P_{N D}$ estimates between the proposed method using CBF-derived $R_{1}$ and gold standard $R_{1}$ are comparable and there is a reduced bias in the estimates.

Figure 5b compares $B P_{N D}$ estimation using 30:60 mins PET data only to the gold standard to demonstrate the need of a CBF-derived $R_{1}$. Due to the lack of data to support the kinetic modelling, the results are noisy and extreme parameter estimates occurred for some regions. These points are beyond the display range in Figure 5b and have skewed the linear regression such that it no longer follows the identity line, and the $95 \%$ CI extends beyond that shown.

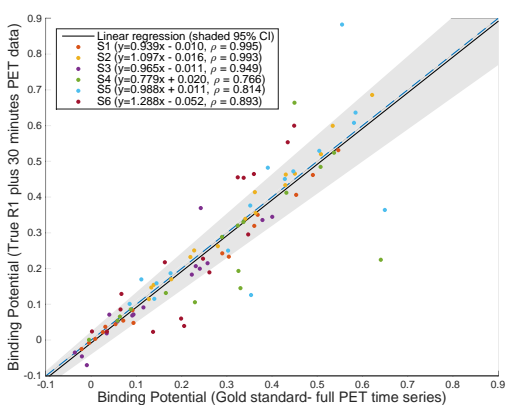

(a) 30:60 mins PET + gold standard $R_{1}$

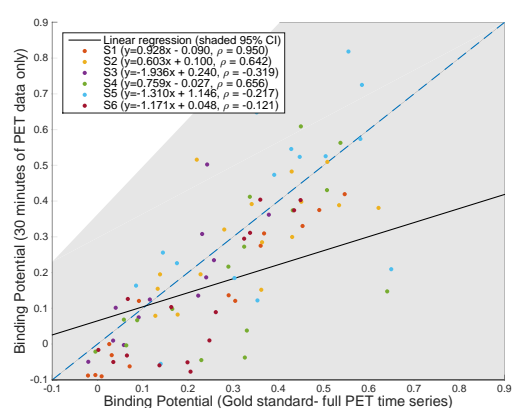

(b) 30:60 mins PET data only

Fig. 5: Estimated binding potential plotted against the gold standard value calculated using full PET time series

\section{Discussion and Conclusion}

This work demonstrates that the proposed method produces estimates of amyloid burden which are comparable to full pharmacokinetic modelling of 0:60-mins $\left[{ }^{18} \mathrm{~F}\right]$ florbetapir PET data, using just 30:60-mins of PET data together with blood flow information from ASL. The proposed method is more accurate than the simplified estimate of amyloid burden, $S U V R-1$, which showed a positive 
bias especially at higher binding potential values. The results of the proposed technique depend on the CBF-derived $R_{1}$ estimate from the ASL data. The ASL data used here were acquired for only 5 minutes without motion correction, and thus susceptible to artefacts and noise. Linear regression between CBF and $R_{1}$ using just 5 subjects could produce errors which may propagate to the binding potential estimation. To reduce the influence of errors in the CBF maps on the parameter estimation, a more complex kinetic model will be explored in future work to penalise the deviation of $R_{1}$ estimation from the CBF-derived $R_{1}$ value. The relationship between CBF from ASL and PET $R_{1}$ will be further explored to tune this regularisation scheme. The application of this technique to PET tracers which bind to other biological targets of interest will also be explored.

Acknowledgements This work was supported by the EPSRC UCL Centre for Doctoral Training in Medical Imaging (EP/L016478/1), UCL Leonard Wolfson Experimental Neurology Centre (PR/ylr/18575), EPSRC (EP/H046410/1, EP/J020990/1, EP/K005278), MRC (MR/J01107X/1), NIHR UCLH Biomedical Research Centre (inc. High Impact Initiative, BW.mn.BRC10269). Insight 1946 receives funding from Alzheimer's Research UK (ARUK-PG2014-1946), MRC Dementia Platform UK (CSUB19166) and The Wolfson Foundation, and support from Avid Radiopharmaceuticals, a wholly owned subsidiary of Eli Lilly. We are grateful to the Insight 46 participants for their involvement in this study.

\section{References}

1. Cselényi, Z., Farde, L.: Quantification of blood flow-dependent component in estimates of beta-amyloid load obtained using quasi-steady-state standardized uptake value ratio. Journal of Cerebral Blood Flow \& Metabolism (March) (2015) 1-9

2. Melbourne, A., Toussaint, N., Owen, D., Simpson, I., Anthopoulos, T., De Vita, E., Atkinson, D., Ourselin, S.: Niftyfit: a software package for multi-parametric modelfitting of 4D magnetic resonance imaging data. Neuroinformatics 14(3) (2016)

3. Lammertsma, A., Hume, S.: Simplified reference tissue model for PET receptor studies. Neuroimage 158(4) (1996) 153-158

4. Klunk, W.E., Engler, H., Nordberg, A., Wang, Y., Blomqvist, G., Holt, D.P., Bergström, M., Savitcheva, I., Huang, G.F., Estrada, S., Ausén, B., Debnath, M.L., Barletta, J., Price, J.C., Sandell, J., Lopresti, B.J., Wall, A., Koivisto, P., Antoni, G., Mathis, C.a., Långström, B.: Imaging Brain Amyloid in Alzheimer's Disease with Pittsburgh Compound-B. Annals of Neurology 55(3) (2004) 306-319

5. Gunn, R.N., Lammertsma, A.A., Hume, S.P., Cunningham, V.J.: Parametric imaging of ligand-receptor binding in PET using a simplified reference region model. NeuroImage 6(4) (1997) 279-287

6. Burgos, N., Cardoso, M.J., Thielemans, K., Modat, M., Dickson, J., Schott, J.M., Atkinson, D., Arridge, S.R., Hutton, B.F., Ourselin, S.: Multi-contrast Attenuation Map Synthesis for PET/MR Scanners: Assessment on FDG and Florbetapir PET Tracers. Eur J of Nuc Med and Mol Imaging 42(9) (2015) 1447-1458

7. Cardoso, M.J., Modat, M., Wolz, R., Melbourne, A., Cash, D., Rueckert, D., Ourselin, S.: Geodesic information flows: spatially-variant graphs and their application to segmentation and fusion. IEEE TMI 99 (2015) 